\title{
JUMPING DEFORMATIONS OF COMPLETE TORIC VARIETIES
}

\author{
HIROSHI SATO
}

Received 30 December 2002

\begin{abstract}
We construct one-parameter complex analytic families whose special fibers are complete toric varieties. Under appropriate assumptions, the general fibers of these families also become toric varieties, and the corresponding fans are explicitly described by the data of the fans associated to the special fibers. Using these families, we construct a deformation family for a certain toric weakened Fano 3-fold. Moreover, we obtain certain examples of toric weakened Fano 4-folds.
\end{abstract}

2000 Mathematics Subject Classification: 14M25, 14J45, 32G05.

1. Introduction. It is well known that the Hirzebruch surface $F_{a}(a \geq 0)$ of degree $a$ is deformed in a one-parameter family to $F_{a-2 k}$, where $k$ is a positive integer such that $a-2 k \geq 0$. In particular, if $a \equiv a^{\prime}(\bmod 2)$, then $F_{a}$ and $F_{a^{\prime}}$ are homeomorphic. In this paper, we generalize this classical result to certain nonsingular complete toric varieties. Namely, for a nonsingular complete toric $d$-fold $V$ which has a toric fibration onto $\mathbf{P}^{1}$ such that its general fiber has at least one symmetric pair of toric prime divisors (see Definition 3.2), we construct a complex analytic family $\left\{V_{t}\right\}_{t \in \mathbb{C}}$ such that $V_{0} \cong V$. Moreover, under appropriate assumptions, the fan corresponding to the general fiber of this family is explicitly described by the data of the fan corresponding to $V$, and $\left\{V_{t}\right\}_{t \neq 0}$ are mutually isomorphic.

As an application of this construction of families, we construct a deformation family for a certain toric weakened Fano 3-fold, that is, a nonsingular toric weak Fano variety which is not Fano but is deformed to a Fano variety. Toric weakened Fano $d$-folds are classified for $d \leq 3$ (see Sato [8]). Moreover, we obtain certain examples of toric weakened Fano 4-folds.

The content of this paper is as follows. In Section 2, we review the homogeneous coordinate of a toric variety, which is a key to our main result. In Section 3, we construct complex analytic families of nonsingular complete varieties over $\mathbb{C}$ as stated above. In Section 4 , as an application of the construction, we study deformations among $\mathbf{P}^{d-1}$-bundles over $\mathbf{P}^{1}$. In Section 5 , we give certain examples of toric weakened Fano 3-folds and 4-folds using the families constructed in Section 3.

2. Homogeneous coordinates of toric varieties. In this section, we recall homogeneous coordinates of toric varieties (see Cox [3] and Oda [6]). 
Let $N=\mathbb{Z}^{d}$ with elements regarded as column vectors, $M:=\operatorname{Hom}_{\mathbb{Z}}(N, \mathbb{Z})$, $N_{\mathbb{R}}:=N \otimes \mathbb{R}, M_{\mathbb{R}}:=M \otimes \mathbb{R}$, and $\Sigma$ a fan in $N$. Throughout this paper, we mean by a cone a nonsingular rational cone and by a fan in $N$ a nonsingular fan which contains at least one $d$-dimensional cone. For $0 \leq i \leq d$, we put $\Sigma(i):=\{\sigma \in$ $\Sigma \mid \operatorname{dim} \sigma=i\}$. Each $\tau \in \Sigma(1)$ determines a unique element $e(\tau) \in N$ which generates the semigroup $\tau \cap N$. Let

$$
G(\Sigma):=\{e(\tau) \in N \mid \tau \in \Sigma(1)\}
$$

and $G(\sigma):=\sigma \cap G(\Sigma)$. We introduce variables $\left\{\mathscr{X}_{\rho} \mid \rho \in G(\Sigma)\right\}$ and consider the polynomial $\operatorname{ring} S:=\mathbb{C}\left[\mathscr{X}_{\rho} \mid \rho \in G(\Sigma)\right]$, which we call the homogeneous coordinate ring of the nonsingular toric $d$-fold $V$ corresponding to $\Sigma$. Let

$$
Z:=\left\{\left(X_{\rho}\right)_{\rho \in G(\Sigma)} \in \mathbb{C}^{G(\Sigma)} \mid \prod_{\rho \in G(\Sigma) \backslash G(\sigma)} X_{\rho}=0 \text { for any } \sigma \in \Sigma\right\} \subset \mathbb{C}^{G(\Sigma)}
$$

On the other hand, by the exact sequence

$$
0 \longrightarrow M \longrightarrow \mathbb{Z}^{G(\Sigma)} \longrightarrow \operatorname{Pic}(V) \longrightarrow 0
$$

we have an exact sequence

$$
1 \longrightarrow G:=\operatorname{Hom}_{\mathbb{Z}}\left(\operatorname{Pic}(V), \mathbb{C}^{\times}\right) \longrightarrow\left(\mathbb{C}^{\times}\right)^{G(\Sigma)} \longrightarrow T_{N} \longrightarrow 1 .
$$

Since $\left(\mathbb{C}^{\times}\right)^{G(\Sigma)}$ acts naturally on $\mathbb{C}^{G(\Sigma)}$, the subgroup $G \subset\left(\mathbb{C}^{\times}\right)^{G(\Sigma)}$ acts on $\mathbb{C}^{G(\Sigma)}$ as

$$
g t:=\left(g\left(\left[D_{\rho}\right]\right) t_{\rho}\right)_{\rho \in G(\Sigma)},
$$

where $g \in G, t=\left(t_{\rho}\right)_{\rho \in G(\Sigma)} \in \mathbb{C}^{G(\Sigma)}$, and $\left[D_{\rho}\right] \in \operatorname{Pic}(V)$ is the class of the toric prime divisor $D_{\rho}$ corresponding to $\rho$. In this setting, the following proposition holds.

Proposition 2.1 (Cox [3, Theorem 2.1]). The subset $\mathbb{C}^{G(\Sigma)} \backslash Z \subset \mathbb{C}^{G(\Sigma)}$ is invariant under the action of $G$, and $V$ is the geometric quotient of $\mathbb{C}^{G(\Sigma)} \backslash Z$ by $G$. The subset $\mathbb{C}^{G(\Sigma)} \backslash Z$ is denoted by $\mathrm{U}(\Sigma)$.

We need the following proposition for this description.

Proposition 2.2 (Cox [3, Theorem 2.1]). For any $\sigma \in \Sigma$,

$$
U_{\sigma} \cong\left(\mathbf{U}(\Sigma)_{\sigma}:=\left\{\left(X_{\rho}\right)_{\rho \in G(\Sigma)} \in \mathbf{U}(\Sigma) \mid \prod_{\rho \in G(\Sigma) \backslash G(\sigma)} X_{\rho} \neq 0\right\}\right) / G
$$

where $U_{\sigma} \subset V$ is the affine toric subvariety associated to $\sigma$. 
3. Constructions of families. In this section, we construct one-parameter complex analytic families whose fibers are nonsingular complete varieties. In particular, the special fibers are nonsingular complete toric varieties. This is a generalization of the classical results on deformations among Hirzebruch surfaces.

Let $\tilde{N}:=\{\mathbf{n} \in N \mid$ the $d$ th coordinate of $\mathbf{n}$ is 0$\}$ and $\tilde{\Sigma}$ a complete fan in $\tilde{N}$. For a complete fan $\Sigma$ in $N$ containing $\tilde{\Sigma}$ as a subfan, we define subfans of $\Sigma$ as follows:

$$
\begin{aligned}
& \Sigma^{+}:=\{\sigma \in \Sigma \mid \text { the } d \text { th coordinate of } \mathbf{n} \text { is nonnegative for any } \mathbf{n} \in \sigma\}, \\
& \Sigma^{-}:=\{\sigma \in \Sigma \mid \text { the } d \text { th coordinate of } \mathbf{n} \text { is nonpositive for any } \mathbf{n} \in \sigma\} .
\end{aligned}
$$

Then, we have $\tilde{\Sigma}=\Sigma^{+} \cap \Sigma^{-}$. We denote by $V$ (resp., $V^{+}$and $V^{-}$) the nonsingular toric $d$-fold corresponding to the fan $\Sigma$ (resp., $\Sigma^{+}$and $\Sigma^{-}$), while we denote by $\tilde{V}$ the nonsingular toric $(d-1)$-fold corresponding to the fan $\tilde{\Sigma}$.

REMARK 3.1. The variety $V$ has a toric fibration $V \rightarrow \mathbf{P}^{1}$ whose general fiber is isomorphic to $\tilde{V}$.

For a nonsingular complete fan $\Sigma$ in $N$, we can define the abstract simplicial complex $\Gamma(\Sigma)$ whose vertex set is $G(\Sigma)$, naturally. We need the following definition.

DEFINITION 3.2. Let $S$ be a nonsingular complete toric variety and $\Sigma_{S}$ the corresponding fan in $N$. Then, a pair $\left(D_{1}, D_{2}\right)$ of distinct toric prime divisors on $S$ is called a symmetric pair if the following conditions hold:

(i) $D_{1}=D_{2}$ in $\operatorname{Pic}(S)$;

(ii) the bijection $\phi: G\left(\Sigma_{S}\right) \rightarrow G\left(\Sigma_{S}\right)$, defined by

$$
\phi\left(\mathbf{n}_{1}\right)=\mathbf{n}_{2}, \quad \phi\left(\mathbf{n}_{2}\right)=\mathbf{n}_{1},\left.\quad \phi\right|_{G(\Sigma) \backslash\left\{\mathbf{n}_{1}, \mathbf{n}_{2}\right\}}=\mathrm{id},
$$

induces the automorphism of $\Gamma\left(\Sigma_{S}\right)$, where $\mathbf{n}_{1}$ and $\mathbf{n}_{2}$ are the elements in $G\left(\Sigma_{S}\right)$ corresponding to $D_{1}$ and $D_{2}$, respectively.

REMARK 3.3. There exists a linear subspace $H \subset N_{\mathbb{R}}$ of codimension 1 such that $G\left(\Sigma_{S}\right) \backslash\left\{\mathbf{n}_{1}, \mathbf{n}_{2}\right\} \subset H$.

EXAMPLE 3.4. The $d$-dimensional projective space $\mathbf{P}^{d}$ has a symmetric pair of toric prime divisors, while a toric bundle over $\mathbf{P}^{1}$ also has a symmetric pair of toric prime divisors.

EXAMPLE 3.5. One can easily check that every nonsingular toric Fano 3-fold has a symmetric pair of toric prime divisors by the classification. There exists a toric Fano 3-fold which is neither $\mathbf{P}^{3}$ nor a toric bundle over $\mathbf{P}^{1}$ (see Batyrev [2] and Watanabe-Watanabe [9]). 
Suppose that $\tilde{V}$ has at least one symmetric pair of toric prime divisors. In the above situation, let

$$
\begin{gathered}
G(\tilde{\Sigma})=\left\{\mathbf{e}_{1}, \ldots, \mathbf{e}_{d-1}, \mathbf{a}_{1}, \ldots, \mathbf{a}_{\rho}\right\}, \\
G\left(\Sigma^{+}\right)=\left\{\mathbf{b}_{1}, \ldots, \mathbf{b}_{m}\right\} \cup G(\widetilde{\Sigma}), \quad G\left(\Sigma^{-}\right)=\left\{\mathbf{c}_{1}, \ldots, \mathbf{c}_{n}\right\} \cup G(\widetilde{\Sigma}),
\end{gathered}
$$

$\left\{\mathbf{e}_{1}, \ldots, \mathbf{e}_{d-1}, \mathbf{b}_{1}\right\}$ the standard basis for $N$, and

$$
\begin{aligned}
& \left(\mathbf{a}_{1}, \ldots, \mathbf{a}_{\rho}, \mathbf{b}_{2}, \ldots, \mathbf{b}_{m}, \mathbf{c}_{1}, \ldots, \mathbf{c}_{n}\right) \\
& =\left(\begin{array}{ccccccccc}
a_{1,1} & \cdots & a_{\rho, 1} & b_{2,1} & \cdots & b_{m, 1} & c_{1,1} & \cdots & c_{n, 1} \\
\vdots & \ddots & \vdots & \vdots & \ddots & \vdots & \vdots & \ddots & \vdots \\
a_{1, d} & \cdots & a_{\rho, d} & b_{2, d} & \cdots & b_{m, d} & c_{1, d} & \cdots & c_{n, d}
\end{array}\right) .
\end{aligned}
$$

Suppose that the pair of the toric prime divisors on $\tilde{V}$ corresponding to $\mathbf{e}_{1}$ and $\mathbf{a}_{1}$ is a symmetric pair. Suppose further that $\left\{\mathbf{e}_{1}, \ldots, \mathbf{e}_{d-1}, \mathbf{b}_{1}\right\}$ generates a $d$-dimensional cone in $\Sigma^{+}$, while $\left\{\mathbf{e}_{1}, \ldots, \mathbf{e}_{d-1}, \mathbf{c}_{1}\right\}$ generates a $d$-dimensional cone in $\Sigma^{-}$. For a nonnegative integer $k$, we construct a complex analytic family.

Since $\left\{\mathbf{a}_{1}, \ldots, \mathbf{a}_{\rho}\right\} \subset G(\tilde{\Sigma})$, we have $a_{1, d}=\cdots=a_{\rho, d}=0$. We have $c_{1, d}=-1$, by the assumption that $\left\{\mathbf{e}_{1}, \ldots, \mathbf{e}_{d-1}, \mathbf{c}_{1}\right\}$ generates a $d$-dimensional cone in $\Sigma^{-}$.

Let $D_{1}, \ldots, D_{d-1} ; A_{1}, \ldots, A_{\rho} ; B_{1}, \ldots, B_{m}$; and $C_{1}, \ldots, C_{n}$ be the toric prime divisors corresponding to $\mathbf{e}_{1}, \ldots, \mathbf{e}_{d-1} ; \mathbf{a}_{1}, \ldots, \mathbf{a}_{\rho} ; \mathbf{b}_{1}, \ldots, \mathbf{b}_{m}$; and $\mathbf{c}_{1}, \ldots, \mathbf{c}_{n}$; respectively. Then, by computing the divisors of the rational functions $\mathbf{e}\left(\mathbf{e}_{1}^{*}\right), \ldots$, $\mathbf{e}\left(\mathbf{e}_{d-1}^{*}\right), \mathbf{e}\left(\mathbf{b}_{1}^{*}\right) \in \mathbb{C}(V)$, where $\left\{\mathbf{e}_{1}^{*}, \ldots, \mathbf{e}_{d-1}^{*}, \mathbf{b}_{1}^{*}\right\} \subset M$ is the dual basis of $\left\{\mathbf{e}_{1}, \ldots\right.$, $\left.\mathbf{e}_{d-1}, \mathbf{b}_{1}\right\}$, we have

$$
\begin{gathered}
D_{1}+a_{1,1} A_{1}+\cdots+a_{\rho, 1} A_{\rho}+b_{2,1} B_{2}+\cdots \\
+b_{m, 1} B_{m}+c_{1,1} C_{1}+\cdots+c_{n, 1} C_{n}=0 \\
D_{2}+a_{1,2} A_{1}+\cdots+a_{\rho, 2} A_{\rho}+b_{2,2} B_{2}+\cdots \\
+b_{m, 2} B_{m}+c_{1,2} C_{1}+\cdots+c_{n, 2} C_{n}=0, \\
\vdots \\
D_{d-1}+a_{1, d-1} A_{1}+\cdots+a_{\rho, d-1} A_{\rho}+b_{2, d-1} B_{2}+\cdots \\
+b_{m, d-1} B_{m}+c_{1, d-1} C_{1}+\cdots+c_{n, d-1} C_{n}=0, \\
B_{1}+b_{2, d} B_{2}+\cdots+b_{m, d} B_{m}-C_{1}+c_{2, d} C_{2}+\cdots+c_{n, d} C_{n}=0
\end{gathered}
$$

in $\operatorname{Pic}(V)$, respectively. Using these equalities, we calculate the homogeneous coordinates of $V, V^{+}, V^{-}$, and $\tilde{V}$.

Let $\left(X_{1}, \ldots, X_{d-1}, Y_{1}, \ldots, Y_{\rho}, Z_{1}, \ldots, Z_{m}, W_{1}, \ldots, W_{n}\right)$ be a homogeneous coordinate of

$$
V \cong \mathbf{U}(\Sigma) / G \text { with } G:=\operatorname{Hom}_{\mathbb{Z}}\left(\operatorname{Pic}(V), \mathbb{C}^{\times}\right)
$$


corresponding to $\mathbf{e}_{1}, \ldots, \mathbf{e}_{d-1} ; \mathbf{a}_{1}, \ldots, \mathbf{a}_{\rho} ; \mathbf{b}_{1}, \ldots, \mathbf{b}_{m}$; and $\mathbf{c}_{1}, \ldots, \mathbf{c}_{n}$; respectively, and $\left(X_{1}^{+}, \ldots, X_{d-1}^{+}, Y_{1}^{+}, \ldots, Y_{\rho}^{+}, Z_{1}^{+}, \ldots, Z_{m}^{+}\right)$a homogeneous coordinate of

$$
V^{+} \cong \mathbf{U}\left(\Sigma^{+}\right) / G^{+} \text {with } G^{+}:=\operatorname{Hom}_{\mathbb{Z}}\left(\operatorname{Pic}\left(V^{+}\right), \mathbb{C}^{\times}\right)
$$

corresponding to $\mathbf{e}_{1}, \ldots, \mathbf{e}_{d-1} ; \mathbf{a}_{1}, \ldots, \mathbf{a}_{\rho} ;$ and $\mathbf{b}_{1}, \ldots, \mathbf{b}_{m}$; respectively. Since $W_{1}, \ldots, W_{n} \neq 0$ on $\bigcup_{\sigma \in \Sigma^{+}} \mathbf{U}(\Sigma)_{\sigma}$, we can define a surjective morphism

$$
\varphi^{+}: \bigcup_{\sigma \in \Sigma^{+}} \mathbf{U}(\Sigma)_{\sigma} \subset \mathbf{U}(\Sigma) \longrightarrow \mathbf{U}\left(\Sigma^{+}\right)
$$

given by

$$
\begin{aligned}
X_{1}^{+} & =X_{1} W_{1}^{c_{1,1}} \cdots W_{n}^{c_{n, 1}}, \ldots, X_{d-1}^{+}=X_{d-1} W_{1}^{c_{1, d-1}} \cdots W_{n}^{c_{n, d-1}}, \\
Y_{1}^{+} & =Y_{1}, \ldots, Y_{\rho}^{+}=Y_{\rho}, \\
Z_{1}^{+} & =Z_{1} W_{1}^{c_{1, d}} \cdots W_{n}^{c_{n, d}}, Z_{2}^{+}=Z_{2}, \ldots, Z_{m}^{+}=Z_{m} .
\end{aligned}
$$

LEMMA 3.6. The morphism $\varphi^{+}$induces an isomorphism

$$
\tilde{\varphi}^{+}:\left(\bigcup_{\sigma \in \Sigma^{+}} \mathbf{U}(\Sigma)_{\sigma}\right) / G \longrightarrow V^{+} .
$$

Proof. By relations (3.5), for $g \in G$, we have

$$
\begin{aligned}
\left(g\left(D_{i}\right) X_{i}\right)\left(g\left(C_{1}\right) W_{1}\right)^{c_{1, i}} \cdots\left(g\left(C_{n}\right) W_{n}\right)^{c_{n, i}} & \\
\quad= & g\left(D_{i}+c_{1, i} C_{1}+\cdots+c_{n, i} C_{n}\right) X_{i} W_{1}^{c_{1, i}} \cdots W_{n}^{c_{n, i}} \\
\quad & =g\left(-\left(a_{1, i} A_{1}+\cdots+a_{\rho, i} A_{\rho}+b_{2, i} B_{2}+\cdots+b_{m, i} B_{m}\right)\right) X_{i} W_{1}^{c_{1, i}} \cdots W_{n}^{c_{n, i}}
\end{aligned}
$$

for any $1 \leq i \leq d-1$, while we have

$$
\begin{aligned}
\left(g\left(B_{1}\right) Z_{1}\right)\left(g\left(C_{1}\right) W_{1}\right)^{c_{1, d}} \cdots\left(g\left(C_{n}\right) W_{n}\right)^{c_{n, d}} & \\
\quad= & g\left(B_{1}+c_{1, d} C_{1}+\cdots+c_{n, d} C_{n}\right) Z_{1} W_{1}^{c_{1, d}} \cdots W_{n}^{c_{n, d}} \\
\quad= & g\left(-\left(b_{2, d} B_{2}+\cdots+b_{m, d} B_{m}\right)\right) Z_{1} W_{1}^{c_{1, d}} \cdots W_{n}^{c_{n, d}} .
\end{aligned}
$$

Therefore, $\varphi^{+}$is compatible with the action of $G$ and $G^{+}$, and hence $\varphi^{+}$induces an isomorphism

$$
\tilde{\varphi}^{+}:\left(\bigcup_{\sigma \in \Sigma^{+}} \mathbf{U}(\Sigma)_{\sigma}\right) / G \subset V \longrightarrow V^{+} \cong \mathbf{U}\left(\Sigma^{+}\right) / G^{+} .
$$

On the other hand, let $\left(X_{1}^{-}, \ldots, X_{d-1}^{-}, Y_{1}^{-}, \ldots, Y_{\rho}^{-}, W_{1}^{-}, \ldots, W_{n}^{-}\right)$be a homogeneous coordinate of $V^{-} \cong \mathbf{U}\left(\Sigma^{-}\right) / G^{-}$with $G^{-}:=\operatorname{Hom}_{\mathbb{Z}}\left(\operatorname{Pic}\left(V^{-}\right), \mathbb{C}^{\times}\right)$corresponding to $\mathbf{e}_{1}, \ldots, \mathbf{e}_{d-1} ; \mathbf{a}_{1}, \ldots, \mathbf{a}_{\rho} ;$ and $\mathbf{c}_{1}, \ldots, \mathbf{c}_{n} ;$ respectively. Similarly as above, 
the surjective morphism

$$
\varphi^{-}: \bigcup_{\sigma \in \Sigma^{-}} \mathbf{U}(\Sigma)_{\sigma} \subset \mathbf{U}(\Sigma) \longrightarrow \mathbf{U}\left(\Sigma^{-}\right)
$$

given by

$$
\begin{gathered}
X_{1}^{-}=X_{1} Z_{1}^{c_{1,1}} Z_{2}^{b_{2, d} c_{1,1}+b_{2,1}} \cdots Z_{m}^{b_{m, d} c_{1,1}+b_{m, 1}}, \ldots, \\
X_{d-1}^{-}=X_{d-1} Z_{1}^{c_{1, d-1}} Z_{2}^{b_{2, d} c_{1, d-1}+b_{2, d-1}} \cdots Z_{m}^{b_{m, d} c_{1, d-1}+b_{m, d-1}}, \\
Y_{1}^{-}=Y_{1}, \ldots, Y_{\rho}^{-}=Y_{\rho}, \quad W_{1}^{-}=Z_{1}^{-1} Z_{2}^{-b_{2, d}} \cdots Z_{m}^{-b_{m, d}} W_{1}, W_{2}^{-}=W_{2}, \ldots, W_{n}^{-}=W_{n},
\end{gathered}
$$

induces an isomorphism

$$
\tilde{\varphi}^{-}:\left(\bigcup_{\sigma \in \Sigma^{-}} \mathbf{U}(\Sigma)_{\sigma}\right) / G \subset V \rightarrow V^{-} \cong \mathbf{U}\left(\Sigma^{-}\right) / G^{-}
$$

Next, let $\left(x_{1}^{+}, \ldots, x_{d-1}^{+}, y_{1}^{+}, \ldots, y_{\rho}^{+}\right)$and $\left(x_{1}^{-}, \ldots, x_{d-1}^{-}, y_{1}^{-}, \ldots, y_{\rho}^{-}\right)$be homogeneous coordinates of $\widetilde{V}$ corresponding to $\mathbf{e}_{1}, \ldots, \mathbf{e}_{d-1}$ and $\mathbf{a}_{1}, \ldots, \mathbf{a}_{\rho}$, respectively, and $z, w \in \mathbb{C}^{\times}$. By similar argument as above, we obtain two isomorphisms

$$
\left(\bigcup_{\sigma \in \tilde{\Sigma}} \mathbf{U}\left(\Sigma^{+}\right)_{\sigma}\right) / G^{+} \subset V^{+} \longrightarrow \tilde{V} \times \mathbb{C}^{\times}, \quad\left(\bigcup_{\sigma \in \tilde{\Sigma}} \mathbf{U}\left(\Sigma^{-}\right)_{\sigma}\right) / G^{-} \subset V^{-} \longrightarrow \tilde{V} \times \mathbb{C}^{\times},
$$

given by

$$
\begin{aligned}
x_{1}^{+} & =X_{1}^{+}\left(Z_{2}^{+}\right)^{b_{2,1}} \cdots\left(Z_{m}^{+}\right)^{b_{m, 1}}, \ldots, x_{d-1}^{+}=X_{d-1}^{+}\left(Z_{2}^{+}\right)^{b_{2, d-1}} \cdots\left(Z_{m}^{+}\right)^{b_{m, d-1}}, \\
y_{1}^{+} & =Y_{1}^{+}, \ldots, y_{\rho}^{+}=Y_{\rho}^{+}, \quad z=Z_{1}^{+}\left(Z_{2}^{+}\right)^{b_{2, d}} \cdots\left(Z_{m}^{+}\right)^{b_{m, d}}, \\
x_{1}^{-} & =X_{1}^{-}\left(W_{2}^{-}\right)^{c_{2,1}+c_{1,1} c_{2, d}} \ldots\left(W_{n}^{-}\right)^{c_{n, 1}+c_{1,1} c_{n, d}}, \ldots \\
x_{d-1}^{-} & =X_{d-1}^{-}\left(W_{2}^{-}\right)^{c_{2, d-1}+c_{1, d-1} c_{2, d}} \cdots\left(W_{n}^{-}\right)^{c_{n, d-1}+c_{1, d-1} c_{n, d}}, \\
y_{1}^{-} & =Y_{1}^{-}, \ldots, y_{\rho}^{-}=Y_{\rho}^{-}, \quad w=W_{1}^{-}\left(W_{2}^{-}\right)^{-c_{2, d}} \cdots\left(W_{n}^{-}\right)^{-c_{n, d}},
\end{aligned}
$$

respectively. These two coordinates of $\tilde{V} \times \mathbb{C}^{\times}$are related as follows:

$$
x_{1}^{+}=x_{1}^{-} w^{c_{1,1}}, \ldots, x_{d-1}^{+}=x_{d-1}^{-} w^{c_{1, d-1}}, \quad y_{1}^{+}=y_{1}^{-}, \ldots, y_{\rho}^{+}=y_{\rho}^{-}, \quad z=\frac{1}{w} .
$$

We construct a one-parameter family of nonsingular complete algebraic varieties parameterized by $t \in \mathbb{C}$ by changing this relation: let $\left\{V_{t}\right\}_{t \in \mathbb{C}}$ be the family we obtain by patching $V^{+}$and $V^{-}$along $\tilde{V} \times \mathbb{C}^{\times}$by the automorphism 


$$
\begin{gathered}
\left(x_{1}^{-}, \ldots, x_{d-1}^{-}, y_{1}^{-}, \ldots, y_{\rho}^{-}, w\right) \mapsto\left(x_{1}^{+}, \ldots, x_{d-1}^{+}, y_{1}^{+}, \ldots, y_{\rho}^{+}, z\right), \text { defined by } \\
x_{1}^{+}=x_{1}^{-} w^{c_{1,1}}+t y_{1}^{-} w^{k}, x_{2}^{+}=x_{2}^{-} w^{c_{1,2}}, \ldots, x_{d-1}^{+}=x_{d-1}^{-} w^{c_{1, d-1}}, \\
y_{1}^{+}=y_{1}^{-}, \ldots, y_{\rho}^{+}=y_{\rho}^{-}, \quad z=\frac{1}{w} .
\end{gathered}
$$

This is well defined since $\left(D_{1}, A_{1}\right)$ is a symmetric pair of toric prime divisors on $\tilde{V}$. Thus, we have the following theorem.

THEOREM 3.7. The family $\left\{V_{t}\right\}_{t \in \mathbb{C}}$ is a complex analytic family whose special fiber $V_{0}$ is isomorphic to $V$.

Finally, we calculate the general fibers of this family under appropriate assumptions. We introduce certain notation.

For any $\mathbf{q}=\left(q_{1}, \ldots, q_{d-1}\right) \in \mathbb{Z}^{d-1}$, we can define a complete fan $\mathbf{q}^{-} \Sigma$ in $N$ as follows:

$$
\mathbf{q}^{-} \Sigma:=\Sigma^{+} \cup\left\{\mathbf{q}^{-} \sigma \mid \sigma \in \Sigma^{-}\right\}
$$

where $\mathbf{q}^{-} \sigma$ is the image of $\sigma$ under the automorphism of $N_{\mathbb{R}}$ corresponding to the matrix

$$
\left(\begin{array}{ccccc}
1 & 0 & \cdots & 0 & q_{1} \\
0 & 1 & \cdots & 0 & q_{2} \\
\vdots & \vdots & \ddots & \vdots & \vdots \\
0 & 0 & \cdots & 1 & q_{d-1} \\
0 & 0 & \cdots & 0 & 1
\end{array}\right)
$$

acting from the left on the elements of $N=\mathbb{Z}^{d}$ regarded as column vectors. We denote by $q^{-} V$ the nonsingular toric $d$-fold corresponding to the fan $q^{-} \sum$.

THEOREM 3.8. For any $t \in \mathbb{C}^{\times}$,

$$
V_{t} \cong\left(2 k,-k a_{1,2}, \ldots,-k a_{1, d-1}\right)^{-} V,
$$

if the following conditions are satisfied:

(i) $m=n=1$,

(ii) $k c_{1, d}+c_{1,1}=c_{1,1}-k \geq 0$.

REMARK 3.9. Theorem 3.8(i) implies that $V$ has a toric bundle structure $V \rightarrow \mathbf{P}^{1}$ whose fiber is isomorphic to $\tilde{V}$.

Proof OF TheOrem 3.8. Let $\left(\hat{x}_{1}^{+}, \ldots, \hat{x}_{d-1}^{+}, \hat{y}_{1}^{+}, \ldots, \hat{y}_{\rho}^{+}, \hat{z}\right)$ be a coordinate of $\tilde{V} \times \mathbb{C}^{\times}$. By assumption, we can define an automorphism $\psi^{+}:\left(x_{1}^{+}, \ldots, x_{d-1}^{+}\right.$, $\left.y_{1}^{+}, \ldots, y_{\rho}^{+}, z\right) \mapsto\left(\hat{x}_{1}^{+}, \ldots, \hat{x}_{d-1}^{+}, \hat{y}_{1}^{+}, \ldots, \hat{y}_{\rho}^{+}, \hat{z}\right)$ of $\tilde{V} \times \mathbb{C}^{\times}$by

$$
\begin{aligned}
& \hat{x}_{1}^{+}:=x_{1}^{+} z^{k}-t y_{1}^{+}, \hat{x}_{2}^{+}:=x_{2}^{+}, \ldots, \hat{x}_{d-1}^{+}:=x_{d-1}^{+}, \\
& \hat{y}_{1}^{+}:=t x_{1}^{+}, \hat{y}_{2}^{+}:=y_{2}^{+}, \ldots, \hat{y}_{\rho}^{+}:=y_{\rho}^{+}, \quad \hat{z}:=z .
\end{aligned}
$$


In fact, we can easily construct the inverse of this morphism. Then, the coordinates $\left(\hat{x}_{1}^{+}, \ldots, \hat{x}_{d-1}^{+}, \hat{y}_{1}^{+}, \ldots, \hat{y}_{\rho}^{+}, \hat{z}\right)$ and $\left(x_{1}^{-}, \ldots, x_{d-1}^{-}, y_{1}^{-}, \ldots, y_{\rho}^{-}, w\right)$ are related as follows:

$$
\begin{aligned}
& \hat{x}_{1}^{+}=\left(x_{1}^{-} w^{c_{1,1}}+t y_{1}^{-} w^{k}\right) w^{-k}-t y_{1}^{-}=x_{1}^{-} w^{c_{1,1}-k}, \\
& \hat{x}_{2}^{+}=x_{2}^{-} w^{c_{1,2}}, \ldots, \hat{x}_{d-1}^{+}=x_{d-1}^{-} w^{c_{1, d-1}}, \\
& \hat{y}_{1}^{+}=t\left(x_{1}^{-} w^{c_{1,1}}+t y_{1}^{-} w^{k}\right), \hat{y}_{2}^{+}=y_{2}^{-}, \ldots, \hat{y}_{\rho}^{+}=y_{\rho}^{-}, \quad \hat{z}=\frac{1}{w} .
\end{aligned}
$$

By considering the action of $\widetilde{G}:=\operatorname{Hom}_{\mathbb{Z}}\left(\operatorname{Pic}(\tilde{V}), \mathbb{C}^{\times}\right)$, these relations are equivalent to

$$
\begin{aligned}
& \hat{x}_{1}^{+}=x_{1}^{-} w^{c_{1,1}-2 k}, \hat{x}_{2}^{+}=x_{2}^{-} w^{c_{1,2}+k a_{1,2}}, \ldots, \hat{x}_{d-1}^{+}=x_{d-1}^{-} w^{c_{1, d-1}+k a_{1, d-1},} \\
& \hat{y}_{1}^{+}=t\left(x_{1}^{-} w^{c_{1,1}-k}+t y_{1}^{-}\right), \hat{y}_{2}^{+}=y_{2}^{-}, \ldots, \hat{y}_{\rho}^{+}=y_{\rho}^{-}, \quad \hat{z}=\frac{1}{w} .
\end{aligned}
$$

Similarly, we can define another automorphism

$$
\psi^{-}:\left(x_{1}^{-}, \ldots, x_{d-1}^{-}, y_{1}^{-}, \ldots, y_{\rho}^{-}, w\right) \longmapsto\left(\hat{x}_{1}^{-}, \ldots, \hat{x}_{d-1}^{-}, \hat{y}_{1}^{-}, \ldots, \hat{y}_{\rho}^{-}, \hat{w}\right)
$$

of $\tilde{V} \times \mathbb{C}^{\times}$by

$$
\begin{gathered}
\hat{x}_{1}^{-}:=x_{1}^{-}, \ldots, \hat{x}_{d-1}^{-}:=x_{d-1}^{-}, \\
\hat{y}_{1}^{-}:=t\left(x_{1}^{-} w^{c_{1,1}-k}+t y_{1}^{-}\right), \hat{y}_{2}^{-}:=y_{2}^{-}, \ldots, \hat{y}_{\rho}^{-}:=y_{\rho}^{-}, \quad \hat{w}:=w .
\end{gathered}
$$

These new coordinates $\left(\hat{x}_{1}^{+}, \ldots, \hat{x}_{d-1}^{+}, \hat{y}_{1}^{+}, \ldots, \hat{y}_{\rho}^{+}, \hat{z}\right)$ and $\left(\hat{x}_{1}^{-}, \ldots, \hat{x}_{d-1}^{-}, \hat{y}_{1}^{-}, \ldots\right.$, $\left.\hat{y}_{\rho}^{-}, \hat{w}\right)$ of $\tilde{V} \times \mathbb{C}^{\times}$are related as follows:

$$
\begin{gathered}
\hat{x}_{1}^{+}=\hat{x}_{1}^{-} \hat{w}^{c_{1,1}-2 k}, \hat{x}_{2}^{+}=\hat{x}_{2}^{-} \hat{w}^{c_{1,2}+k a_{1,2}}, \ldots, \hat{x}_{d-1}^{+}=\hat{x}_{d-1}^{-} \hat{w}^{c_{1, d-1}+k a_{1, d-1}}, \\
\hat{y}_{1}^{+}=\hat{y}_{1}^{-}, \ldots, \hat{y}_{\rho}^{+}=\hat{y}_{\rho}^{-}, \quad \hat{z}=\frac{1}{\hat{w}} .
\end{gathered}
$$

We show that the automorphisms $\psi^{+}$and $\psi^{-}$are extended to the automorphisms of $V^{+}$and $V^{-}$, respectively. Let

$$
\begin{aligned}
& \hat{X}_{1}^{+}:=X_{1}^{+}\left(Z_{1}^{+}\right)^{k}-t Y_{1}^{+}, \hat{X}_{2}^{+}:=X_{2}^{+}, \ldots, \hat{X}_{d-1}^{+}:=X_{d-1}^{+}, \\
& \hat{Y}_{1}^{+}:=t X_{1}^{+}, \hat{Y}_{2}^{+}:=Y_{2}^{+}, \ldots, \hat{Y}_{\rho}^{+}:=Y_{\rho}^{+}, \quad \hat{Z}_{1}^{+}:=Z_{1}^{+} .
\end{aligned}
$$

By the assumptions $m=1$ and $\left(D_{1}, A_{1}\right)$ is a symmetric pair of toric prime divisors on $\tilde{V}$, this defines an automorphism $\left(X_{1}^{+}, \ldots, X_{d-1}^{+}, Y_{1}^{+}, \ldots, Y_{\rho}^{+}, Z_{1}^{+}\right) \mapsto$ $\left(\hat{X}_{1}^{+}, \ldots, \hat{X}_{d-1}^{+}, \hat{Y}_{1}^{+}, \ldots, \hat{Y}_{\rho}^{+}, \hat{Z}_{1}^{+}\right)$of $V^{+}$whose restriction to $\tilde{V} \times \mathbb{C}^{\times}$is $\psi^{+}$. Similarly, 
by the assumption $k c_{1, d}+c_{1,1} \geq 0$, by putting

$$
\begin{gathered}
\hat{X}_{1}^{-}:=X_{1}^{-}, \ldots, \hat{X}_{d-1}^{-}:=X_{d-1}^{-}, \\
\hat{Y}_{1}^{-}:=t X_{1}^{-}\left(W_{1}^{-}\right)^{k c_{1, d}+c_{1,1}}, \hat{Y}_{2}^{-}:=Y_{2}^{-}, \ldots, \hat{Y}_{\rho}^{-}:=Y_{\rho}^{-}, \quad \hat{W}_{1}^{-}:=W_{1}^{-},
\end{gathered}
$$

we obtain an automorphism $\left(X_{1}^{-}, \ldots, X_{d-1}^{-}, Y_{1}^{-}, \ldots, Y_{\rho}^{-}, W_{1}^{-}\right) \mapsto\left(\hat{X}_{1}^{-}, \ldots, \hat{X}_{d-1}^{-}\right.$, $\left.\hat{Y}_{1}^{-}, \ldots, \hat{Y}_{\rho}^{-}, \hat{W}_{1}^{-}\right)$of $V^{-}$whose restriction to $\tilde{V} \times \mathbb{C}^{\times}$is $\psi^{-}$.

On the other hand, let $\mathbf{c}_{1}^{\prime}$ be the element in $G\left(\mathbf{q}^{-} \Sigma\right)$ corresponding to $\mathbf{c}_{1}$, where $\mathbf{q}=\left(2 k,-k a_{1,2}, \ldots,-k a_{1, d-1}\right)$. Then,

$$
\mathbf{c}_{1}^{\prime}=\left(\begin{array}{c}
c_{1,1}+2 k c_{1, d} \\
c_{1,2}-k a_{1,2} c_{1, d} \\
\vdots \\
c_{1, d-1}-k a_{1, d-1} c_{1, d} \\
c_{1, d}
\end{array}\right)
$$

On $V^{-}$, equalities (3.5) are

$$
\begin{gathered}
D_{1}+a_{1,1} A_{1}+\cdots+a_{\rho, 1} A_{\rho}+c_{1,1} C_{1}=0, \\
D_{2}+a_{1,2} A_{1}+\cdots+a_{\rho, 2} A_{\rho}+c_{1,2} C_{1}=0, \\
\vdots \\
D_{d-1}+a_{1, d-1} A_{1}+\cdots+a_{\rho, d-1} A_{\rho}+c_{1, d-1} C_{1}=0, \\
-C_{1}=0
\end{gathered}
$$

in $\operatorname{Pic}\left(V^{-}\right)$. Obviously, these equalities are equivalent to

$$
\begin{gathered}
D_{1}+a_{1,1} A_{1}+\cdots+a_{\rho, 1} A_{\rho}+\left(c_{1,1}+2 k c_{1, d}\right) C_{1}=0, \\
D_{2}+a_{1,2} A_{1}+\cdots+a_{\rho, 2} A_{\rho}+\left(c_{1,2}-k a_{1,2} c_{1, d}\right) C_{1}=0, \\
\vdots \\
D_{d-1}+a_{1, d-1} A_{1}+\cdots+a_{\rho, d-1} A_{\rho}+\left(c_{1, d-1}-k a_{1, d-1} c_{1, d}\right) C_{1}=0, \\
-C_{1}=0
\end{gathered}
$$

in $\operatorname{Pic}\left(V^{-}\right)$. Therefore, the action of $G^{-}$on $\mathrm{U}\left(\Sigma^{-}\right)$coincides with the action of $G^{\prime}:=\operatorname{Hom}_{\mathbb{Z}}\left(\operatorname{Pic}\left(V^{\prime}\right), \mathbb{C}^{\times}\right)$on $\mathbf{U}\left(\Sigma^{\prime}\right)$, where

$$
\Sigma^{\prime}=\left(\mathbf{q}^{-} \Sigma \backslash \Sigma^{+}\right) \cup \tilde{\Sigma}
$$

and $V^{\prime}$ is the toric $d$-fold corresponding to $\Sigma^{\prime}$. 
Thus, for any $t \in \mathbb{C}^{\times}$, we have

$$
V_{t} \cong\left(2 k,-k a_{1,2}, \ldots,-k a_{1, d-1}\right)^{-} V
$$

4. Projective space bundles over the projective line. The classical results for deformations among Hirzebruch surfaces are well known. As a generalization of this results, for $\mathbf{P}^{2}$-bundles over $\mathbf{P}^{1}$, Nakamura [5] showed the following proposition.

Proposition 4.1 (Nakamura [5]). For integers $a, b, c, a^{\prime}, b^{\prime}, c^{\prime}$, let

$$
V=\mathbf{P}_{\mathbf{P}^{1}}(\mathcal{O}(a) \oplus \mathscr{O}(b) \oplus \mathscr{O}(c)), \quad V^{\prime}=\mathbf{P}_{\mathbf{P}^{1}}\left(\mathscr{O}\left(a^{\prime}\right) \oplus \mathscr{O}\left(b^{\prime}\right) \oplus \mathscr{O}\left(c^{\prime}\right)\right) .
$$

Then the following are equivalent:

(i) $a+b+c \equiv a^{\prime}+b^{\prime}+c^{\prime}(\bmod 3)$;

(ii) there exist $\mathbf{P}^{2}$-bundles $V_{0}, \ldots, V_{m}$ over $\mathbf{P}^{1}$ such that $V_{0} \cong V, V_{m} \cong V^{\prime}$, and $V_{i-1}$ is deformed to $V_{i}$ for any $1 \leq i \leq m$;

(iii) $V$ and $V^{\prime}$ are homeomorphic.

We generalize the implication (i) $\Rightarrow$ (ii) of Proposition 4.1 for $\mathbf{P}^{d-1}$-bundles over $\mathbf{P}^{1}$ using the one-parameter families constructed in Theorem 3.7. Harris [4] studied this case. For fundamental properties of primitive collections and primitive relations, see Batyrev [1, 2] and Sato [7]. We use the notation as in Section 3.

Let $V$ be a $\mathbf{P}^{d-1}$-bundle over $\mathbf{P}^{1}$, that is,

$$
V=V\left(p_{1}, \ldots, p_{d-1}\right):=\mathbf{P}_{\mathbf{P}^{1}}\left(\mathcal{O} \oplus \mathcal{O}\left(p_{1}\right) \oplus \cdots \oplus \mathcal{O}\left(p_{d-1}\right)\right),
$$

where $p_{1}, \ldots, p_{d-1}$ are nonnegative integers. Then, the primitive relations of the corresponding fan $\Sigma$ are

$$
\mathbf{e}_{1}+\cdots+\mathbf{e}_{d-1}+\mathbf{a}_{1}=0, \quad \mathbf{b}_{1}+\mathbf{c}_{1}=p_{1} \mathbf{e}_{1}+\cdots+p_{d-1} \mathbf{e}_{d-1},
$$

where $G(\Sigma)=\left\{\mathbf{e}_{1}, \ldots, \mathbf{e}_{d-1}, \mathbf{a}_{1}, \mathbf{b}_{1}, \mathbf{c}_{1}\right\}$. For a nonnegative integer $k$ such that $a_{1}-k \geq 0$, the conditions in Theorem 3.8 are satisfied. Therefore, there exists a one-parameter complex analytic family $\left\{V_{t}\right\}_{t \in \mathbb{C}}$ such that

$$
V_{t} \cong \begin{cases}V & \text { if } t=0, \\ (2 k, k, \ldots, k)^{-} V & \text { if } t \neq 0 .\end{cases}
$$

We show that for $V\left(p_{1}, \ldots, p_{d-1}\right)$ and $V\left(p_{1}^{\prime}, \ldots, p_{d-1}^{\prime}\right)$, if $p_{1}+\cdots+p_{d-1} \equiv p_{1}^{\prime}+$ $\cdots+p_{d-1}^{\prime}(\bmod d)$, then there exist nonsingular toric $d$-folds $V_{0}, \ldots, V_{m}$ such that each $V_{i}$ is a $\mathbf{P}^{d-1}$-bundle over $\mathbf{P}^{1}, V_{0} \cong V\left(p_{1}, \ldots, p_{d-1}\right), V_{m} \cong V\left(p_{1}^{\prime}, \ldots, p_{d-1}^{\prime}\right)$, and $V_{i-1}$ is deformed by a one-parameter family to $V_{i}$ for any $1 \leq i \leq m$. 
Let $k=1$. Suppose that there exists $1 \leq i \leq d-1$ such that $p_{i} \geq 2$. So, we may assume that $p_{1} \geq p_{2} \geq \cdots \geq p_{l}>p_{l+1}=\cdots=p_{d-1}=0$ by changing the order of the indices, where $p_{1} \geq 2$. Then, by the family (4.4), $V$ is deformed to $(2,1, \ldots, 1)^{-} V$. The primitive relations of $(2,1, \ldots, 1)^{-} \Sigma$ are

$$
\begin{gathered}
\mathbf{e}_{1}+\cdots+\mathbf{e}_{d-1}+\mathbf{a}_{1}=0, \\
\mathbf{b}_{1}+\mathbb{C}_{1}^{\prime}=\left(\begin{array}{c}
p_{1}-2 \\
p_{2}-1 \\
\vdots \\
p_{l}-1 \\
p_{l+1}-1 \\
\vdots \\
p_{d-1}-1 \\
0
\end{array}\right)= \begin{cases}\left(p_{1}-1\right) \mathbf{e}_{1}+p_{2} \mathbf{e}_{2}+\cdots+p_{l} \mathbf{e}_{l}+\mathbf{a}_{1} & \text { if } l<d-1, \\
\left(p_{1}-2\right) \mathbf{e}_{1}+\left(p_{2}-1\right) \mathbf{e}_{2}+\cdots+\left(p_{l}-1\right) \mathbf{e}_{l} & \text { if } l=d-1,\end{cases} \\
\end{gathered}
$$

where $G\left((2,1, \ldots, 1)^{-} \Sigma\right)=\left\{\mathbf{e}_{1}, \ldots, \mathbf{e}_{d-1}, \mathbf{a}_{1}, \mathbf{b}_{1}, \mathbb{C}_{1}^{\prime}\right\}$. We can replace $V$ by $(2,1, \ldots, 1)^{-} V$ and carry out this operation again. This operation terminates in finite steps, and $V$ becomes $V\left(p_{1}, \ldots, p_{d-1}\right)$ such that $p_{1} \leq 1, \ldots, p_{d-1} \leq 1$. In each step, $p_{1}+\cdots+p_{d-1} \in \mathbb{Z} / d \mathbb{Z}$ does not change. Thus, we have the following proposition.

Proposition 4.2. For integers $a_{1}, \ldots, a_{d}, a_{1}^{\prime}, \ldots, a_{d}^{\prime}$, let $V=\mathbf{P}_{\mathbf{P}^{1}}\left(\mathcal{O}\left(a_{1}\right) \oplus \cdots \oplus\right.$ $\left.\mathcal{O}\left(a_{d}\right)\right)$ and $V^{\prime}=\mathbf{P}_{\mathbf{p}^{1}}\left(\mathcal{O}\left(a_{1}^{\prime}\right) \oplus \cdots \oplus \mathcal{O}\left(a_{d}^{\prime}\right)\right)$. If $a_{1}+\cdots+a_{d} \equiv a_{1}^{\prime}+\cdots+a_{d}^{\prime}(\bmod d)$, then there exist $\mathbf{P}^{d-1}$-bundles $V_{0}, \ldots, V_{m}$ over $\mathbf{P}^{1}$ such that $V_{0} \cong V, V_{m} \cong V^{\prime}$, and $V_{i-1}$ is deformed to $V_{i}$ for any $1 \leq i \leq m$. In particular, $V$ and $V^{\prime}$ are homeomorphic.

5. Weakened Fano varieties. The following definition is important for the birational geometry.

DEFINITION 5.1. Let $V$ be a nonsingular projective variety. Then, $V$ is called a Fano (resp., weak Fano) variety if its anticanonical divisor $-K_{V}$ is ample (resp., nef and big).

The following definition was proposed by Minagawa in connection with "Reid's fantasy" for weak Fano 3-folds.

DEFINITION 5.2. Let $V$ be a nonsingular weak Fano variety over $\mathbb{C}$ and $\Delta_{\epsilon}:=$ $\{t \in \mathbb{C}|| t \mid<\epsilon\}$ for a sufficiently small real number $\epsilon>0$. Then, $V$ is called a weakened Fano variety if $V$ is not a nonsingular Fano variety and there exists a small deformation $\varphi: \mathscr{V} \rightarrow \Delta_{\epsilon}$ such that $\mathscr{V}_{0}:=\varphi^{-1}(0) \cong V$, while $\mathscr{V}_{t}:=\varphi^{-1}(t)$ is a nonsingular Fano variety for any $t \in \Delta_{\epsilon} \backslash\{0\}$. 
In this section, we give a deformation family for a certain toric weakened Fano 3-fold using the families constructed in Section 3. Toric weakened Fano 3-folds are completely classified by Sato [8]. Moreover, we obtain nine examples of toric weakened Fano 4-folds. We use the notation as in Section 3.

EXAMPLE 5.3. Let $V$ be the nonsingular toric weakened Fano 3 -fold of type $X_{0}^{3}$ in the sense of Sato [8], that is, the primitive relations of $\Sigma$ are

$$
\mathbf{e}_{1}+\mathbf{a}_{1}=\mathbf{e}_{2}, \quad \mathbf{e}_{2}+\mathbf{a}_{2}=0, \quad \mathbf{b}_{1}+\mathbb{C}_{1}=2 \mathbf{e}_{1},
$$

where $G(\Sigma)=\left\{\mathbf{e}_{1}, \mathbf{e}_{2}, \mathbf{a}_{1}, \mathbf{a}_{2}, \mathbf{b}_{1}, \mathbb{C}_{1}\right\}$. The variety $V$ is an $F_{1}$-bundle over $\mathbf{P}^{1}$, where $F_{1}$ is the Hirzebruch surface of degree 1 . Therefore, by Theorems 3.7 and 3.8, there exists a complex analytic family $\left\{V_{t}\right\}_{t \in \mathbb{C}}$ such that

$$
V_{0} \cong V
$$

while

$$
V_{t} \cong(2,-1)^{-} V \quad(t \neq 0)
$$

The primitive relations of $(2,-1)^{-} \sum$ are

$$
\mathbf{e}_{1}+\mathbf{a}_{1}=\mathbf{e}_{2}, \quad \mathbf{e}_{2}+\mathbf{a}_{2}=0, \quad \mathbf{b}_{1}+\mathbb{C}_{1}^{\prime}=\mathbf{e}_{2},
$$

where $G(\Sigma)=\left\{\mathbf{e}_{1}, \mathbf{e}_{2}, \mathbf{a}_{1}, \mathbf{a}_{2}, \mathbf{b}_{1}, \mathbb{C}_{1}^{\prime}\right\}$. The toric Fano 3 -fold we want is $(2,-1)^{-} V$ (see $[8$, Section 4]).

In the same way as in Example 5.3, we obtain certain examples of toric weakened Fano 4-folds which does not decompose into direct products of lowerdimensional varieties. In the following, $G(\Sigma)=\left\{\mathbf{n}_{1}, \mathbf{n}_{2}, \ldots\right\}$ and the fans corresponding to toric weakened Fano 4-folds are described in terms of primitive relations. We also give the types of general fibers. The symbols of types of nonsingular toric Fano 4-folds are those of Batyrev [2] and Sato [7]:

(i) $\mathbf{n}_{1}+\mathbf{n}_{4}=\mathbf{n}_{2}, \mathbf{n}_{2}+\mathbf{n}_{3}+\mathbf{n}_{5}=0$, and $\mathbf{n}_{6}+\mathbf{n}_{7}=2 \mathbf{n}_{1}$ (type $D_{7}$ );

(ii) $\mathbf{n}_{1}+\mathbf{n}_{4}=\mathbf{n}_{2}, \mathbf{n}_{2}+\mathbf{n}_{6}=0, \mathbf{n}_{3}+\mathbf{n}_{5}=\mathbf{n}_{2}$, and $\mathbf{n}_{7}+\mathbf{n}_{8}=2 \mathbf{n}_{1}$ (type $L_{1}$ );

(iii) $\mathbf{n}_{1}+\mathbf{n}_{4}=\mathbf{n}_{2}, \mathbf{n}_{2}+\mathbf{n}_{6}=0, \mathbf{n}_{3}+\mathbf{n}_{5}=\mathbf{n}_{6}$, and $\mathbf{n}_{7}+\mathbf{n}_{8}=2 \mathbf{n}_{1}$ (type $L_{13}$ );

(iv) $\mathbf{n}_{1}+\mathbf{n}_{4}=\mathbf{n}_{2}, \mathbf{n}_{2}+\mathbf{n}_{5}=\mathbf{n}_{3}, \mathbf{n}_{3}+\mathbf{n}_{6}=0$, and $\mathbf{n}_{7}+\mathbf{n}_{8}=2 \mathbf{n}_{1}$ (type $L_{2}$ );

(v) $\mathbf{n}_{5}+\mathbf{n}_{6}=0, \mathbf{n}_{3}+\mathbf{n}_{7}=0, \mathbf{n}_{2}+\mathbf{n}_{3}=\mathbf{n}_{5}, \mathbf{n}_{5}+\mathbf{n}_{7}=\mathbf{n}_{2}, \mathbf{n}_{2}+\mathbf{n}_{6}=\mathbf{n}_{7}, \mathbf{n}_{1}+\mathbf{n}_{4}=$ $\mathbf{n}_{2}$, and $\mathbf{n}_{8}+\mathbf{n}_{9}=2 \mathbf{n}_{1}$ (type $\left.Q_{1}\right)$;

(vi) $\mathbf{n}_{5}+\mathbf{n}_{6}=0, \mathbf{n}_{3}+\mathbf{n}_{7}=0, \mathbf{n}_{2}+\mathbf{n}_{3}=\mathbf{n}_{5}, \mathbf{n}_{5}+\mathbf{n}_{7}=\mathbf{n}_{2}, \mathbf{n}_{2}+\mathbf{n}_{6}=\mathbf{n}_{7}, \mathbf{n}_{1}+\mathbf{n}_{4}=$ $\mathbf{n}_{3}$, and $\mathbf{n}_{8}+\mathbf{n}_{9}=2 \mathbf{n}_{1}$ (type $Q_{13}$ );

(vii) $\mathbf{n}_{5}+\mathbf{n}_{6}=0, \mathbf{n}_{3}+\mathbf{n}_{7}=0, \mathbf{n}_{2}+\mathbf{n}_{3}=\mathbf{n}_{5}, \mathbf{n}_{5}+\mathbf{n}_{7}=\mathbf{n}_{2}, \mathbf{n}_{2}+\mathbf{n}_{6}=\mathbf{n}_{7}, \mathbf{n}_{1}+\mathbf{n}_{4}=$ $\mathbf{n}_{5}$, and $\mathbf{n}_{8}+\mathbf{n}_{9}=2 \mathbf{n}_{1}$ (type $Q_{8}$ );

(viii) $\mathbf{n}_{5}+\mathbf{n}_{6}=0, \mathbf{n}_{3}+\mathbf{n}_{7}=0, \mathbf{n}_{2}+\mathbf{n}_{3}=\mathbf{n}_{5}, \mathbf{n}_{5}+\mathbf{n}_{7}=\mathbf{n}_{2}, \mathbf{n}_{2}+\mathbf{n}_{6}=\mathbf{n}_{7}, \mathbf{n}_{1}+\mathbf{n}_{4}=$ 0 , and $\mathbf{n}_{8}+\mathbf{n}_{9}=2 \mathbf{n}_{1}$ (type $Q_{11}$ ); 
(ix) $\mathbf{n}_{5}+\mathbf{n}_{8}=0, \mathbf{n}_{2}+\mathbf{n}_{5}=\mathbf{n}_{3}, \mathbf{n}_{3}+\mathbf{n}_{8}=\mathbf{n}_{2}, \mathbf{n}_{3}+\mathbf{n}_{6}=\mathbf{n}_{5}, \mathbf{n}_{3}+\mathbf{n}_{7}=0, \mathbf{n}_{2}+\mathbf{n}_{6}=$ $0, \mathbf{n}_{6}+\mathbf{n}_{8}=\mathbf{n}_{7}, \mathbf{n}_{2}+\mathbf{n}_{7}=\mathbf{n}_{8}, \mathbf{n}_{5}+\mathbf{n}_{7}=\mathbf{n}_{6}, \mathbf{n}_{1}+\mathbf{n}_{4}=\mathbf{n}_{2}$, and $\mathbf{n}_{9}+\mathbf{n}_{10}=2 \mathbf{n}_{1}$ (type $U_{1}$ ).

ACKNOWLEDGments. This work was partially supported by the Grant-inAid for Japan Society for the Promotion of Science (JSPS) Fellows, The Ministry of Education, Science, Sports, and Culture, Japan. The author wishes to thank Professors Tadao Oda, Masa-Nori Ishida, Tadashi Ashikaga, Takeshi Kajiwara, Yasuhiro Nakagawa, and Tatsuhiro Minagawa for advice and encouragement.

\section{REFERENCES}

[1] V. V. Batyrev, On the classification of smooth projective toric varieties, Tôhoku Math. J. (2) 43 (1991), no. 4, 569-585.

[2] On the classification of toric Fano 4-folds, J. Math. Sci. (New York) 94 (1999), no. 1, 1021-1050.

[3] D. A. Cox, The homogeneous coordinate ring of a toric variety, J. Algebraic Geom. 4 (1995), no. 1, 17-50.

[4] J. Harris, A bound on the geometric genus of projective varieties, Ann. Scuola Norm. Sup. Pisa Cl. Sci. (4) 8 (1981), no. 1, 35-68.

[5] I. Nakamura, Global deformations of $\mathbf{P}^{2}$-bundles over $\mathbf{P}^{1}$, J. Math. Kyoto Univ. 38 (1998), no. 1, 29-54.

[6] T. Oda, Convex Bodies and Algebraic Geometry-An Introduction to the Theory of Toric Varieties, Ergebnisse der Mathematik und ihrer Grenzgebiete (3), vol. 15, Springer-Verlag, Berlin, 1988.

[7] H. Sato, Toward the classification of higher-dimensional toric Fano varieties, Tôhoku Math. J. (2) 52 (2000), no. 3, 383-413.

[8] , The classification of smooth toric weakened Fano 3-folds, Manuscripta Math. 109 (2002), no. 1, 73-84.

[9] K. Watanabe and M. Watanabe, The classification of Fano 3-folds with torus embeddings, Tokyo J. Math. 5 (1982), no. 1, 37-48.

Hiroshi Sato: Department of Mathematics, Graduate School of Science and Engineering, Tokyo Institute of Technology, 2-12-1 Oh-okayama, Meguro-ku, Tokyo 152-8551, Japan

E-mail address: hi rosato@math.titech.ac.jp 


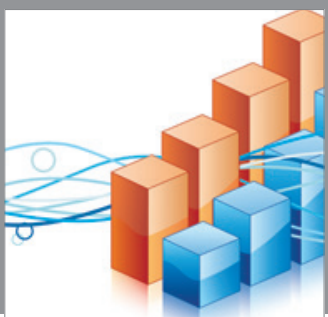

Advances in

Operations Research

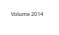

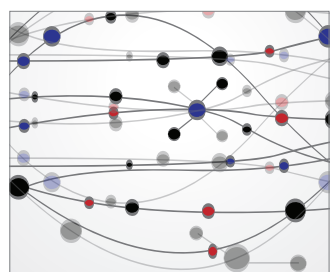

\section{The Scientific} World Journal
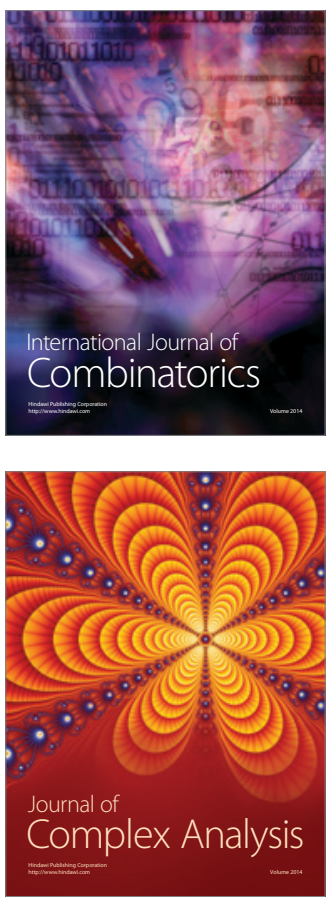

International Journal of

Mathematics and

Mathematical

Sciences
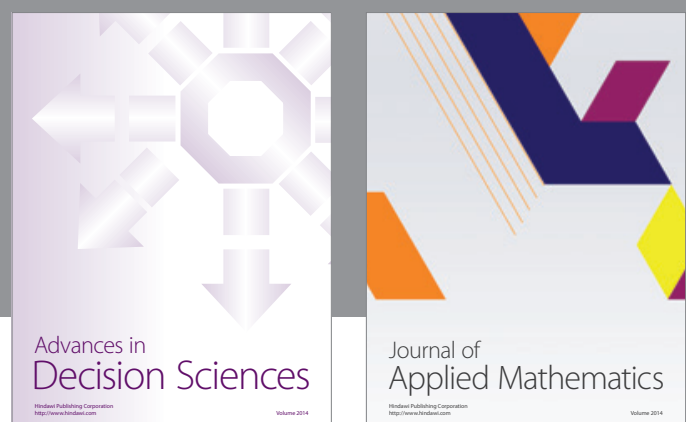

Journal of

Applied Mathematics
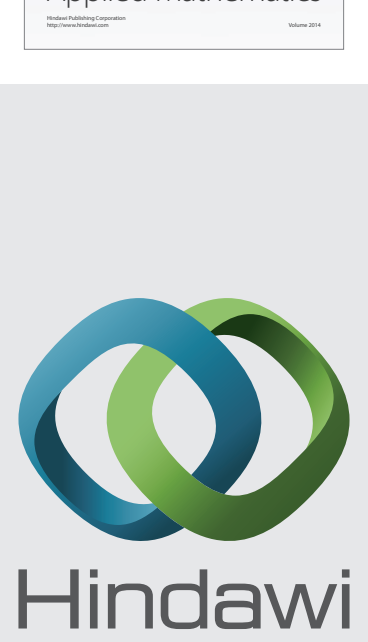

Submit your manuscripts at http://www.hindawi.com
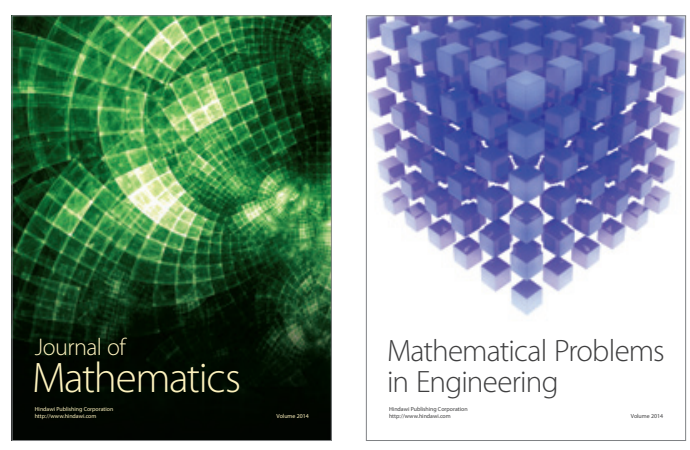

Mathematical Problems in Engineering
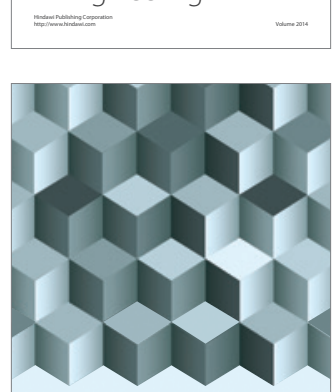

Journal of

Function Spaces
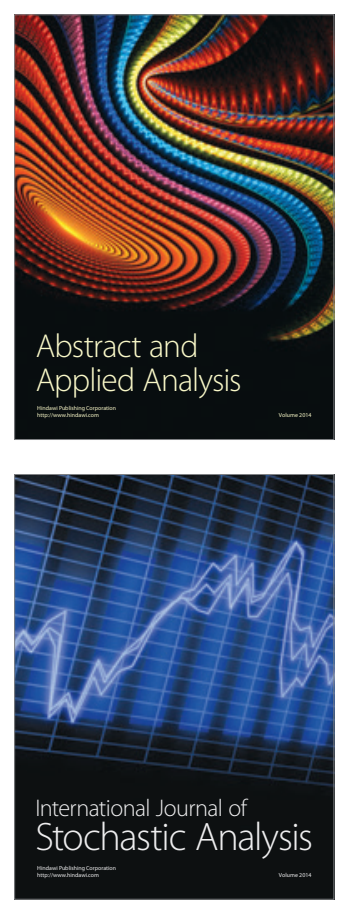

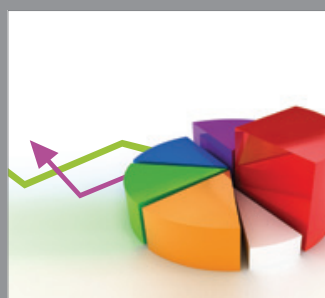

ournal of

Probability and Statistics

Promensencen
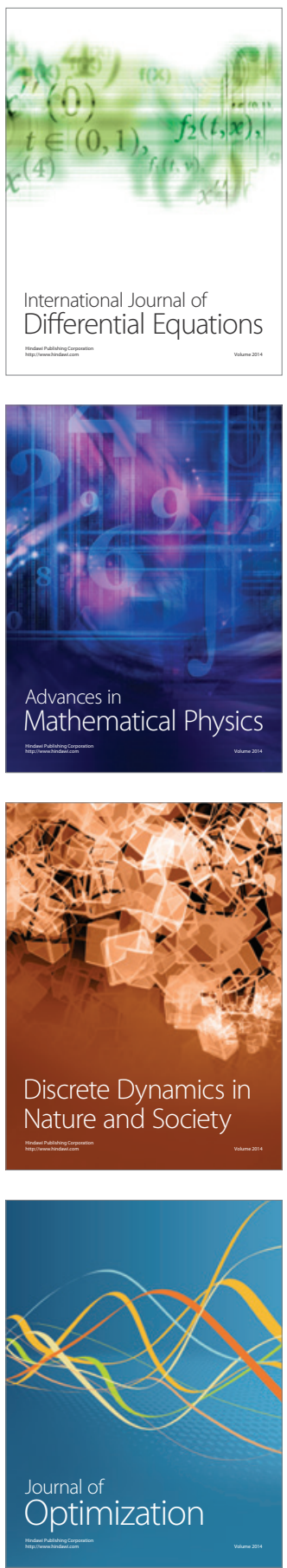